Article

\title{
Some Fractional Hermite-Hadamard Type Inequalities for Interval-Valued Functions
}

\author{
Fangfang Shi ${ }^{1}$, Guoju Ye ${ }^{1, *}$, Dafang Zhao ${ }^{2}$ and Wei Liu ${ }^{1}$ \\ 1 College of Science, Hohai University, Nanjing 210098, China; 15298387799@163.com (F.S.); \\ liuw626@hhu.edu.cn (W.L.) \\ 2 School of Mathematics and Statistics, Hubei Normal University, Huangshi 435002, China; \\ dafangzhao@163.com \\ * Correspondence: yegj@hhu.edu.cn; Tel.: +86-152-9838-7799
}

Received: 6 February 2020; Accepted: 22 March 2020; Published: 4 April 2020

check for updates

\begin{abstract}
In this paper, firstly we prove the relationship between interval $h$-convex functions and interval harmonically $h$-convex functions. Secondly, several new Hermite-Hadamard type inequalities for interval $h$-convex functions via interval Riemann-Liouville type fractional integrals are established. Finally, we obtain some new fractional Hadamard-Hermite type inequalities for interval harmonically $h$-convex functions by using the above relationship. Also we discuss the importance of our results and some special cases. Our results extend and improve some previously known results.
\end{abstract}

Keywords: Hermite-Hadamard type inequalities; interval-valued functions; fractional integrals

\section{Introduction}

Hermite-Hadamard inequality was firstly discovered by Hermite and Hadamard for convex functions are considerable significant in the literature. Since Hermite-Hadamard inequality has been regarded as one of the most useful inequalities in mathematical analysis and optimization, many papers have provided generalizations, refinements and extensions, see [1-4]. Due to the fractional integral has played a irreplaceable part in various scientific fields and importance of Hermite-Hadamard type inequalities, Sarikaya et al. [5] presented Hermite-Hadamard type inequalities via fractional integrals. Moreover, many papers relating to fractional integral inequalities have been obtained for different classes of functions, see [6-8].

On the other hand, interval analysis was initially developed as an attempt to deal with interval uncertainty that appears in computer graphics [9], automatic error analysis [10], and many others. Recently, several authors have extended their research by combining integral inequalities with interval-valued functions (IVFs), one can see Chalco-Cano et al. [11], Román-Flores et al. [12], Flores-Franulič et al. [13], Zhao et al. [14,15], An et al. [16]. As a further extension, more and more Hermite-Hadamard type inequalities involving interval Riemann-Liouville type fractional integral have been obtained for different classes of IVFs, see for interval convex functions [17], for interval harmonically convex functions [18] and the references therein.

Motivated by the ongoing research, We proved the relationship between interval $h$-convex functions and interval harmonically $h$-convex functions, then we establish some new Hermite-Hadamard type inequalities for interval $h$-convex functions and interval harmonically $h$-convex functions via interval Riemann-Liouville type fractional integrals. Our results extend and improve some known results. Also we discuss the importance of our results and some special cases. In addition, results obtained in this paper may be extended for other classes of convex functions 
including interval $\left(h_{1}, h_{2}\right)$-convex functions and interval Log-h-convex functions and used as a tool to investigate the research of optimization and probability, among others.

\section{Preliminaries and Result}

Let us denote by $\mathbb{R}_{\mathcal{I}}$ the collection of all nonempty closed intervals of the real line $\mathbb{R}$. We call $[z]=[\underline{z}, \bar{z}]$ positive if $\underline{z}>0$. We denote by $\mathbb{R}_{\mathcal{I}}^{+}$and $\mathbb{R}^{+}$the set of all positive intervals and the set of all positive numbers of $\mathbb{R}$, respectively. For $[z]=[\underline{z}, \bar{z}],[s]=[\underline{s}, \bar{s}] \in \mathbb{R}_{\mathcal{I}}$, the inclusion " $\subseteq$ " is defined by

$$
[\underline{z}, \bar{z}] \subseteq[\underline{s}, \bar{s}] \Leftrightarrow \underline{s} \leq \underline{z}, \bar{z} \leq \bar{s} .
$$

For $\lambda \in \mathbb{R}$, the Minkowski addition and scalar multiplication are defined by

$$
\begin{gathered}
{[z]+[s]=[\underline{z}, \bar{z}]+[\underline{s}, \bar{s}]=[\underline{z}+\underline{s}, \bar{z}+\bar{s}]} \\
\lambda[z]=\lambda[\underline{z}, \bar{z}]=\left\{\begin{array}{cc}
{[\lambda \underline{z}, \lambda \bar{z}],} & \lambda>0 \\
\{0\}, & \lambda=0 \\
{[\lambda \bar{z}, \lambda \underline{z}],} & \lambda<0
\end{array}\right.
\end{gathered}
$$

respectively. The conception of Riemann integral for interval-valued function is introduced in [19]. Moreover, we have

Definition 1. [19] Let $f:[a, b] \rightarrow \mathbb{R}_{\mathcal{I}}$ be an interval-valued function such that $f=[f, \bar{f}]$. Then the $f$ is Riemann integrable on $[a, b]$ iff $f$ and $\bar{f}$ are Riemann integrable on $[a, b]$ and

$$
\int_{a}^{b} f(t) d t=\left[\int_{a}^{b} f(t) d t, \int_{a}^{b} \bar{f}(t) d t\right]
$$

The set of all Riemann integrable IVFs on $[a, b]$ will be denoted by $\mathcal{I} \mathcal{R}_{([a, b])}$. For more basic notations with interval analysis, see [19,20]. Furthermore, we recall the following results in [17].

Definition 2. Let $f:[a, b] \rightarrow \mathbb{R}_{\mathcal{I}}$ be an interval-valued function and $f \in \mathcal{I R}_{([a, b])}$. Then the interval Riemann-Liouville type fractional integrals of $f$ are defined by

$$
\mathcal{J}_{a^{+}}^{\alpha} f(t)=\frac{1}{\Gamma(\alpha)} \int_{a}^{t}(t-v)^{\alpha-1} f(v) d v, \quad t>a .
$$

and

$$
\mathcal{J}_{b^{-}}^{\alpha} f(t)=\frac{1}{\Gamma(\alpha)} \int_{t}^{b}(v-t)^{\alpha-1} f(v) d v, \quad t<b .
$$

where $\alpha>0$ and $\Gamma$ is the Gamma function.

Definition 3. [14] Let $h:[0,1] \rightarrow \mathbb{R}^{+}$be a non-negative function. We say that $f:[a, b] \rightarrow \mathbb{R}_{\mathcal{I}}^{+}$is interval $h$-convex function or that $f \in S X\left(h,[a, b], \mathbb{R}_{\mathcal{I}}^{+}\right)$, if for all $x, y \in[a, b]$ and $v \in[0,1]$, we have

$$
f(v x+(1-v) y) \supseteq h(v) f(x)+h(1-v) f(y) .
$$

Definition 4. [15] Let $h:[0,1] \rightarrow \mathbb{R}^{+}$be a non-negative function. We say that $f:[a, b] \rightarrow \mathbb{R}_{\mathcal{I}}^{+}$is interval harmonically h-convex function or that $f \in \operatorname{SHX}\left(h,[a, b], \mathbb{R}_{\mathcal{I}}^{+}\right)$, if for all $x, y \in[a, b]$ and $v \in[0,1]$, we have

$$
f\left(\frac{x y}{v x+(1-v) y}\right) \supseteq h(1-v) f(x)+h(v) f(y) .
$$


Next, we will present the relationship between interval $h$-convex functions and interval harmonically $h$-convex functions which will be used in Section 4 .

Theorem 1. $f(x) \in \operatorname{SHX}\left(h,[a, b], \mathbb{R}_{\mathcal{I}}^{+}\right)$iff $f\left(\frac{1}{x}\right) \in S X\left(h,[a, b], \mathbb{R}_{\mathcal{I}}^{+}\right)$.

Proof. Let $\psi(x)=f\left(\frac{1}{x}\right)$. Since $f \in \operatorname{SHX}\left(h,[a, b], \mathbb{R}_{\mathcal{I}}^{+}\right)$,

$$
f\left(\frac{x y}{v x+(1-v) y}\right) \supseteq h(1-v) f(x)+h(v) f(y) .
$$

By using $A=\frac{1}{x}$ and $B=\frac{1}{y}$ to replace $x$ and $y$, respectively, applying (1)

$$
\begin{aligned}
f\left(\frac{\frac{1}{x y}}{v \frac{1}{x}+(1-v) \frac{1}{y}}\right) & =f\left(\frac{1}{(1-v) x+v y}\right) \\
& =\psi((1-v) x+v y) \\
& \supseteq h(v) f\left(\frac{1}{y}\right)+h(1-v) f\left(\frac{1}{x}\right) \\
& =h(v) \psi(y)+h(1-v) \psi(x),
\end{aligned}
$$

which gives that $\psi \in S X\left(h,[a, b], \mathbb{R}_{\mathcal{I}}^{+}\right)$.

On the other hand, if $\psi \in S X\left(h,[a, b], \mathbb{R}_{\mathcal{I}}^{+}\right)$, then

$$
\psi(v x+(1-v) y) \supseteq h(v) \psi(x)+h(1-v) \psi(y) .
$$

In the same way as above, we have

$$
\begin{aligned}
\psi\left(v \frac{1}{x}+(1-v) \frac{1}{y}\right) & =f\left(\frac{1}{v \frac{1}{x}+(1-v) \frac{1}{y}}\right)=f\left(\frac{x y}{(1-v) x+v y}\right) \\
& \supseteq h(v) \psi\left(\frac{1}{x}\right)+h(1-v) \psi\left(\frac{1}{y}\right) \\
& =h(v) f(x)+h(1-v) f(y),
\end{aligned}
$$

which gives that $f \in \operatorname{SHX}\left(h,[a, b], \mathbb{R}_{\mathcal{I}}^{+}\right)$. We have completed the proof.

Remark 1. If $h(v)=v$ and $f=\bar{f}$, then we get the Lemma 2.1 of [6].

\section{Fractional Hermite-Hadamard Type Inequalities of Interval $h$-Convex Functions}

In this section, we will prove some new Hermite-Hadamard type inequalities for interval $h$-convex functions via interval Riemann-Liouville type integrals.

Theorem 2. Let $f:[a, b] \rightarrow \mathbb{R}_{\mathcal{I}}^{+}$be an interval-valued function such that $f=[\underline{f}, \bar{f}]$ and $f \in \mathcal{I R}_{([a, b])}, h$ : $[0,1] \rightarrow \mathbb{R}^{+}$be a non-negative function and $h\left(\frac{1}{2}\right) \neq 0$. If $f \in S X\left(h,[a, b], \mathbb{R}_{\mathcal{I}}^{+}\right)$, then

$$
\begin{aligned}
\frac{1}{\alpha h\left(\frac{1}{2}\right)} f\left(\frac{a+b}{2}\right) & \supseteq \frac{\Gamma(\alpha)}{(b-a)^{\alpha}}\left[\mathcal{J}_{a^{+}}^{\alpha} f(b)+\mathcal{J}_{b^{-}}^{\alpha} f(a)\right] \\
& \supseteq[f(a)+f(b)] \int_{0}^{1} v^{\alpha-1}[h(v)+h(1-v)] d v
\end{aligned}
$$

with $\alpha>0$. 
Proof. Since $f \in S X\left(h,[a, b], \mathbb{R}_{\mathcal{I}}^{+}\right)$, we have

$$
\frac{1}{h\left(\frac{1}{2}\right)} f\left(\frac{x+y}{2}\right) \supseteq f(x)+f(y) .
$$

Let $x=v a+(1-v) b, y=(1-v) a+v b, v \in[0,1]$, then

$$
\frac{1}{h\left(\frac{1}{2}\right)} f\left(\frac{a+b}{2}\right) \supseteq f(v a+(1-v) b)+f((1-v) a+v b) .
$$

Multiplying both sides (3) by $v^{\alpha-1}$ and integrating on $[0,1]$, we get

$$
\begin{aligned}
& \frac{1}{\alpha h\left(\frac{1}{2}\right)} f\left(\frac{a+b}{2}\right) \\
= & \frac{1}{h\left(\frac{1}{2}\right)} f\left(\frac{a+b}{2}\right) \int_{0}^{1} v^{\alpha-1} d v \\
\supseteq & {\left[\int_{0}^{1} v^{\alpha-1} f(v a+(1-v) b) d v+\int_{0}^{1} v^{\alpha-1} f((1-v) a+v b) d v\right] } \\
= & {\left[\int_{0}^{1} v^{\alpha-1}(\underline{f}(v a+(1-v) b)+\underline{f}((1-v) a+v b)) d v,\right.} \\
& \left.\int_{0}^{1} v^{\alpha-1}(\bar{f}(v a+(1-v) b)+\bar{f}((1-v) a+v b)) d v\right] \\
= & {\left[\int_{b}^{a}(\tau(\mu))^{\alpha-1} \underline{f}(\mu) \frac{d \mu}{a-b}+\int_{a}^{b}(1-\tau(\mu))^{\alpha-1} \underline{f}(\mu) \frac{d \mu}{b-a^{\prime}}\right.} \\
= & \frac{\Gamma(\alpha)}{(b-a)^{\alpha}}\left[J_{a^{+}}^{\alpha} \underline{f}(b)+J_{b^{-}}^{\alpha} \underline{f}(a), J_{a^{+}}^{\alpha} \bar{f}(b)+J_{b^{-}}^{\alpha} \bar{f}(a)\right] \\
= & \frac{\Gamma(\alpha)}{(b-a)^{\alpha}}\left[\mathcal{J}_{a^{+}}^{\alpha} f(b)+\mathcal{J}_{b^{-}}^{\alpha} f(a)\right] .
\end{aligned}
$$

where $\tau(\mu)=\frac{b-\mu}{b-a}$.

Similarly, since $f \in S X\left(h,[a, b], \mathbb{R}_{\mathcal{I}}^{+}\right)$,

$$
f(v a+(1-v) b)+f((1-v) a+v b) \supseteq[h(v)+h(1-v)][f(a)+f(b)] .
$$

Multiplying both sides (5) by $v^{\alpha-1}$ and integrating on $[0,1]$, we have

$$
\frac{\Gamma(\alpha)}{(b-a)^{\alpha}}\left[\mathcal{J}_{a^{+}}^{\alpha} f(b)+\mathcal{J}_{b^{-}}^{\alpha} f(a)\right] \supseteq[f(a)+f(b)] \int_{0}^{1} v^{\alpha-1}[h(v)+h(1-v)] d v .
$$

By combining (4) with (6), and the result follows.

Example 1. Suppose that $[a, b]=[1,2]$. Let $h(v)=v$ for all $v \in[0,1]$ and $\alpha=\frac{1}{2}, f:[a, b] \rightarrow \mathbb{R}_{\mathcal{I}}^{+}$be defined by

$$
f(t)=[-\sqrt{t}+2, \sqrt{t}+2] .
$$

We obtain

$$
\frac{1}{\alpha h\left(\frac{1}{2}\right)} f\left(\frac{a+b}{2}\right)=[8-2 \sqrt{6}, 8+2 \sqrt{6}]
$$

$\frac{\Gamma(\alpha)}{(b-a)^{\alpha}}\left[\mathcal{J}_{a^{+}}^{\alpha} f(b)+\mathcal{J}_{b^{-}}^{\alpha} f(a)\right]=\left[7-\sqrt{2}-\frac{\pi}{2}-\log (\sqrt{2}+1), 9+\sqrt{2}+\frac{\pi}{2}+\log (\sqrt{2}+1)\right]$, 


$$
[f(a)+f(b)] \int_{0}^{1} v^{\alpha-1}[h(v)+h(1-v)] d v=[6-2 \sqrt{2}, 6+2 \sqrt{2}] .
$$

Then we get

$$
[8-2 \sqrt{6}, 8+2 \sqrt{6}] \supseteq\left[7-\sqrt{2}-\frac{\pi}{2}-\log (\sqrt{2}+1), 9+\sqrt{2}+\frac{\pi}{2}+\log (\sqrt{2}+1)\right] \supseteq[6-2 \sqrt{2}, 6+2 \sqrt{2}] .
$$

Consequently, Theorem 2 is verified.

Remark 2. If $\alpha=1$, then we get Theorem 4.1 of [14]. If $h(v)=v$, then we get Theorem 2.5 of [17]. If $f=\bar{f}$ and $\alpha=1$, then we get Theorem 6 of [4]. If $f=\bar{f}$ and $h(v)=v$, then we get Theorem 2 of [5].

Theorem 3. Let $f, g:[a, b] \rightarrow \mathbb{R}_{\mathcal{I}}^{+}$be two interval-valued functions such that $f=[f, \bar{f}], g=[\underline{g}, \bar{g}]$ and $f g \in \mathcal{I R}_{([a, b])}, h_{1}, h_{2}:[0,1] \rightarrow \mathbb{R}^{+}$be non-negative functions. If $f \in S X\left(h_{1},[a, b], \mathbb{R}_{\mathcal{I}}^{+}\right), \bar{g} \in$ $\operatorname{SX}\left(h_{2},[a, b], \mathbb{R}_{\mathcal{I}}^{+}\right)$, then

$$
\begin{aligned}
& \frac{\Gamma(\alpha)}{(b-a)^{\alpha}}\left[\mathcal{J}_{a^{+}}^{\alpha} f(b) g(b)+\mathcal{J}_{b^{-}}^{\alpha} f(a) g(a)\right] \\
\supseteq & \mathcal{M}(a, b) \int_{0}^{1} v^{\alpha-1}\left[h_{1}(v) h_{2}(v)+h_{1}(1-v) h_{2}(1-v)\right] d v \\
& +\mathcal{N}(a, b) \int_{0}^{1} v^{\alpha-1}\left[h_{1}(v) h_{2}(1-v)+h_{1}(1-v) h_{2}(v)\right] d v
\end{aligned}
$$

where

$$
\mathcal{M}(a, b)=f(a) g(a)+f(b) g(b), \mathcal{N}(a, b)=f(a) g(b)+f(b) g(a) .
$$

Proof. By hypothesis, one has

$$
\begin{aligned}
& f(v a+(1-v) b) \supseteq h_{1}(v) f(a)+h_{1}(1-v) f(b), \\
& g(v a+(1-v) b) \supseteq h_{2}(v) g(a)+h_{2}(1-v) g(b) .
\end{aligned}
$$

Since $f, g \in \mathbb{R}_{\mathcal{I}}^{+}$, we obtain

$$
\begin{aligned}
& f(v a+(1-v) b) g(v a+(1-v) b) \\
\supseteq & h_{1}(v) h_{2}(v) f(a) g(a)+h_{1}(1-v) h_{2}(1-v) f(b) g(b) \\
& +h_{1}(v) h_{2}(1-v) f(a) g(b)+h_{1}(1-v) h_{2}(v) f(b) g(a) .
\end{aligned}
$$

In the same way as above, we have

$$
\begin{aligned}
& f((1-v) a+v b) g((1-v) a+v b) \\
\supseteq & h_{1}(1-v) h_{2}(1-v) f(a) g(a)+h_{1}(v) h_{2}(v) f(b) g(b) \\
\quad+ & h_{1}(1-v) h_{2}(v) f(a) g(b)+h_{1}(v) h_{2}(1-v) f(b) g(a) .
\end{aligned}
$$

By adding (8) and (9), we obtain

$$
\begin{aligned}
& f(v a+(1-v) b) g(v a+(1-v) b)+f((1-v) a+v b) g((1-v) a+v b) \\
\supseteq & {\left[h_{1}(v) f(a)+h_{1}(1-v) f(b)\right]\left[h_{2}(v) g(a)+h_{2}(1-v) g(b)\right] } \\
& +\left[h_{1}(1-v) f(a)+h_{1}(v) f(b)\right]\left[h_{2}(1-v) g(a)+h_{2}(v) g(b)\right] \\
= & \mathcal{M}(a, b)\left[h_{1}(v) h_{2}(v)+h_{1}(1-v) h_{2}(1-v)\right] \\
& +\mathcal{N}(a, b)\left[h_{1}(1-v) h_{2}(v)+h_{1}(v) h_{2}(1-v)\right] .
\end{aligned}
$$


Multiplying both sides (10) by $v^{\alpha-1}$ and integrating on $[0,1]$, we have

$$
\begin{aligned}
& \int_{0}^{1} v^{\alpha-1} f(v a+(1-v) b) g(v a+(1-v) b) d v \\
& +\int_{0}^{1} v^{\alpha-1} f((1-v) a+v b) g((1-v) a+v b) d v \\
\supseteq & \mathcal{M}(a, b) \int_{0}^{1} v^{\alpha-1}\left[h_{1}(v) h_{2}(v)+h_{1}(1-v) h_{2}(1-v)\right] d v \\
& +\mathcal{N}(a, b) \int_{0}^{1} v^{\alpha-1}\left[h_{1}(1-v) h_{2}(v)+h_{1}(v) h_{2}(1-v)\right] d v .
\end{aligned}
$$

By Definition 2, we obtain

$$
\begin{aligned}
& \int_{0}^{1} v^{\alpha-1} f(v a+(1-v) b) g(v a+(1-v) b) d v=\frac{\Gamma(\alpha)}{(b-a)^{\alpha}} \mathcal{J}_{a^{+}}^{\alpha} f(b) g(b), \\
& \int_{0}^{1} v^{\alpha-1} f((1-v) a+v b) g((1-v) a+v b) d v=\frac{\Gamma(\alpha)}{(b-a)^{\alpha}} \mathcal{J}_{b^{-}}^{\alpha} f(a) g(a) .
\end{aligned}
$$

By substituting the equalities (12) and (13) in (11), then we have inequality (7).

Remark 3. If $h(v)=v$, then we get Theorem 3.5 of [17]. If $\alpha=1$, then we get Theorem 4.5 of [14]. If $f=\bar{f}$ and $\alpha=1$, then we get Theorem 7 of [4].

Theorem 4. Let $f, g:[a, b] \rightarrow \mathbb{R}_{\mathcal{I}}^{+}$be two interval-valued functions such that $f=[\underline{f}, \bar{f}], g=[\underline{g}, \bar{g}]$ and $f g \in$ $\mathcal{I R}_{([a, b])}, h_{1}, h_{2}:[0,1] \rightarrow \mathbb{R}^{+}$be non-negative functions and $h_{1}\left(\frac{1}{2}\right) h_{2}\left(\frac{1}{2}\right) \neq 0$. If $f \in S X\left(h_{1},[\bar{a}, b], \mathbb{R}_{\mathcal{I}}^{+}\right), g \in$ $\operatorname{SX}\left(h_{2},[a, b], \mathbb{R}_{\mathcal{I}}^{+}\right)$, then

$$
\begin{aligned}
& \frac{1}{\alpha h_{1}\left(\frac{1}{2}\right) h_{2}\left(\frac{1}{2}\right)} f\left(\frac{a+b}{2}\right) g\left(\frac{a+b}{2}\right) \\
\supseteq & \frac{\Gamma(\alpha)}{(b-a)^{\alpha}}\left[\mathcal{J}_{a^{+}}^{\alpha} f(b) g(b)+\mathcal{J}_{b^{-}}^{\alpha} f(a) g(a)\right] \\
& +\mathcal{M}(a, b) \int_{0}^{1}\left[v^{\alpha-1}+(1-v)^{\alpha-1}\right] h_{1}(v) h_{2}(1-v) d v \\
& +\mathcal{N}(a, b) \int_{0}^{1}\left[v^{\alpha-1}+(1-v)^{\alpha-1}\right] h_{1}(1-v) h_{2}(1-v) d v .
\end{aligned}
$$

Proof. Since $f \in S X\left(h_{1},[a, b], \mathbb{R}_{\mathcal{I}}^{+}\right), g \in S X\left(h_{2},[a, b], \mathbb{R}_{\mathcal{I}}^{+}\right)$, we get

$$
\begin{aligned}
& f\left(\frac{a+b}{2}\right) g\left(\frac{a+b}{2}\right) \\
= & f\left(\frac{v a+(1-v) b}{2}+\frac{(1-v) a+v b}{2}\right) g\left(\frac{v a+(1-v) b}{2}+\frac{(1-v) a+v b}{2}\right) \\
\supseteq & h_{1}\left(\frac{1}{2}\right) h_{2}\left(\frac{1}{2}\right)[f(v a+(1-v) b)+f((1-v) a+v b)][q(v a+(1-v) b)+q((1-v) a+v b)] \\
\supseteq & h_{1}\left(\frac{1}{2}\right) h_{2}\left(\frac{1}{2}\right)[f(v a+(1-v) b) g(v a+(1-v) b)+f((1-v) a+v b) g((1-v) a+v b)] \\
& +h_{1}\left(\frac{1}{2}\right) h_{2}\left(\frac{1}{2}\right)\left[\left(h_{1}(v) h_{2}(1-v)+h_{1}(1-v) h_{2}(v)\right) \mathcal{M}(a, b)\right. \\
& \left.+\left(h_{1}(v) h_{2}(v)+h_{1}(1-v) h_{2}(1-v)\right) \mathcal{N}(a, b)\right] .
\end{aligned}
$$

Multiplying both sides (15) by $v^{\alpha-1}$ and integrating on $[0,1]$, we have inequality (14). 
Remark 4. If $h(v)=v$, then we get Theorem 3.6 of [17]. If $\alpha=1$, then we get Theorem 4.6 of [14]. If $f \underline{f}=\bar{f}$ and $\alpha=1$, then we get Theorem 8 of [4].

\section{Fractional Hermite-Hadamard Type Inequalities of Interval Harmonically $h$-Convex Functions}

In this section, we will use the above results to get some Hermite-Hadamard type inequalities for interval harmonically $h$-convex functions via interval Riemann-Liouville type integrals and some special cases are also discussed.

Theorem 5. Let $f:[a, b] \rightarrow \mathbb{R}_{\mathcal{I}}^{+}$be an interval-valued function such that $f=[f, \bar{f}]$ and $f \in \mathcal{I R}_{([a, b]),} h:$ $[0,1] \rightarrow \mathbb{R}^{+}$be a non-negative function and $h\left(\frac{1}{2}\right) \neq 0$. If $f \in \operatorname{SHX}\left(h,[a, b], \mathbb{R}_{\mathcal{I}}^{+}\right)$, then

$$
\begin{aligned}
& \frac{1}{\alpha h\left(\frac{1}{2}\right)} f\left(\frac{2 a b}{a+b}\right) \\
\supseteq & \Gamma(\alpha)\left(\frac{a b}{b-a}\right)^{\alpha}\left[\mathcal{J}_{\left(\frac{1}{a}\right)^{-}}^{\alpha}(f \circ \xi)\left(\frac{1}{b}\right)+\mathcal{J}_{\left(\frac{1}{b}\right)^{+}}^{\alpha}(f \circ \xi)\left(\frac{1}{a}\right)\right] \\
\supseteq & {[f(a)+f(b)] \int_{0}^{1} v^{\alpha-1}[h(v)+h(1-v)] d v }
\end{aligned}
$$

where $\xi(x)=\frac{1}{x}$.

Proof. Let $\psi(x)=f\left(\frac{1}{x}\right)$. By Theorem 1 , we have $\psi \in S X\left(h,[a, b], \mathbb{R}_{\mathcal{I}}^{+}\right)$and

$$
\frac{1}{h\left(\frac{1}{2}\right)} \psi\left(\frac{x+y}{2 x y}\right) \supseteq \psi\left(\frac{1}{x}\right)+\psi\left(\frac{1}{y}\right) \text {. }
$$

Let $x=\frac{a b}{v a+(1-v) b}, y=\frac{a b}{(1-v) a+v b}, v \in[0,1]$. Then

$$
\frac{1}{h\left(\frac{1}{2}\right)} \psi\left(\frac{a+b}{2 a b}\right) \supseteq \psi\left(\frac{v a+(1-v) b}{a b}\right)+\psi\left(\frac{(1-v) a+v b}{a b}\right),
$$

Multiplying both sides (17) by $v^{\alpha-1}$ and integrating on $[0,1]$, we have

$$
\begin{aligned}
& \frac{1}{\alpha h\left(\frac{1}{2}\right)} \psi\left(\frac{a+b}{2 a b}\right) \\
\supseteq & {\left[\int_{0}^{1} v^{\alpha-1} \psi\left(\frac{v a+(1-v) b}{a b}\right) d v+\int_{0}^{1} v^{\alpha-1} \psi\left(\frac{(1-v) a+v b}{a b}\right) d v\right] } \\
= & \Gamma(\alpha)\left(\frac{a b}{b-a}\right)^{\alpha}\left[\mathcal{J}_{\left(\frac{1}{a}\right)^{-}}^{\alpha} \psi\left(\frac{1}{b}\right)+\mathcal{J}_{\left(\frac{1}{b}\right)^{+}}^{\alpha} \psi\left(\frac{1}{a}\right)\right]
\end{aligned}
$$

Similarly, we have

$$
\begin{aligned}
& \psi\left(\frac{v a+(1-v) b}{a b}\right) \supseteq h(v) \psi\left(\frac{1}{b}\right)+h(1-v) \psi\left(\frac{1}{a}\right), \\
& \psi\left(\frac{(1-v) a+v b}{a b}\right) \supseteq h(v) \psi\left(\frac{1}{a}\right)+h(1-v) \psi\left(\frac{1}{b}\right) .
\end{aligned}
$$


Then,

$$
\psi\left(\frac{v a+(1-v) b}{a b}\right)+\psi\left(\frac{(1-v) a+v b}{a b}\right) \supseteq[h(v)+h(1-v)]\left[\psi\left(\frac{1}{a}\right)+\psi\left(\frac{1}{b}\right)\right] .
$$

Multiplying both sides (19) by $v^{\alpha-1}$ and integrating on $[0,1]$, we have

$$
\begin{aligned}
& \Gamma(\alpha)\left(\frac{a b}{b-a}\right)^{\alpha}\left[\mathcal{J}_{\left(\frac{1}{a}\right)^{-}}^{\alpha} \psi\left(\frac{1}{b}\right)+\mathcal{J}_{\left(\frac{1}{b}\right)^{+}}^{\alpha} \psi\left(\frac{1}{a}\right)\right] \\
\supseteq & {\left[\psi\left(\frac{1}{a}\right)+\psi\left(\frac{1}{b}\right)\right] \int_{0}^{1} v^{\alpha-1}[h(v)+h(1-v)] d t . }
\end{aligned}
$$

By (18) and (20), we have inequality (16).

Remark 5. If $h(v)=v$, then we get Theorem 3.6 of [18]. If $\alpha=1$, then we get Theorem 2 of [15]. If $\underline{f}=$ $\bar{f}$ and $\alpha=1$, then we get Theorem 3.2 of [3]. If $\underline{f}=\bar{f}$ and $h(v)=v$, then we get Theorem 4 of [7].

Theorem 6. Let $f, g:[a, b] \rightarrow \mathbb{R}_{\mathcal{I}}^{+}$be two interval-valued functions such that $f=[f, \bar{f}], g=[\underline{g}, \bar{g}]$ and $f g \in \mathcal{I R}_{([a, b])}, h_{1}, h_{2}:[0,1] \rightarrow \mathbb{R}^{+}$be non-negative functions. If $f \in \operatorname{SHX}\left(h_{1},[a, b], \mathbb{R}_{\mathcal{I}}^{+}\right), \bar{g} \in$ $\operatorname{SHX}\left(h_{2},[a, b], \mathbb{R}_{\mathcal{I}}^{+}\right)$, then

$$
\begin{aligned}
& \Gamma(\alpha)\left(\frac{a b}{b-a}\right)^{\alpha}\left[\mathcal{J}_{\left(\frac{1}{b}\right)^{+}}^{\alpha}(f \circ \xi)\left(\frac{1}{a}\right)(g \circ \xi)\left(\frac{1}{a}\right)+\mathcal{J}_{\left(\frac{1}{a}\right)^{-}}^{\alpha}(f \circ \xi)\left(\frac{1}{b}\right)(g \circ \xi)\left(\frac{1}{b}\right)\right] \\
& \supseteq \mathcal{M}(a, b) \int_{0}^{1}\left[v^{\alpha-1}+(1-v)^{\alpha-1}\right] h_{1}(v) h_{2}(v) d v \\
& \quad+\mathcal{N}(a, b) \int_{0}^{1}\left[v^{\alpha-1}+(1-v)^{\alpha-1}\right] h_{1}(v) h_{2}(1-v) d v
\end{aligned}
$$

where $\xi(x)=\frac{1}{x}$.

Proof. The proof is completed by combining Theorems 1, 3 and 5 .

Remark 6. If $h_{1}(v)=h_{2}(v)=v$, then

$$
\begin{aligned}
& \Gamma(\alpha)\left(\frac{a b}{b-a}\right)^{\alpha}\left[\mathcal{J}_{\left(\frac{1}{b}\right)^{+}}^{\alpha}(f \circ \xi)\left(\frac{1}{a}\right)(g \circ \xi)\left(\frac{1}{a}\right)+\mathcal{J}_{\left(\frac{1}{a}\right)^{-}}^{\alpha}(f \circ \xi)\left(\frac{1}{b}\right)(g \circ \xi)\left(\frac{1}{b}\right)\right] \\
& \supseteq \mathcal{M}(a, b) \int_{0}^{1} v^{2}\left[v^{\alpha-1}+(1-v)^{\alpha-1}\right] d v+\mathcal{N}(a, b) \int_{0}^{1} v(1-v)\left[v^{\alpha-1}+(1-v)^{\alpha-1}\right] d v .
\end{aligned}
$$

If $\alpha=1$, then we get Theorem 4 of [15]. If $f=\bar{f}$ and $\alpha=1$, then we get Theorem 3.6 of [3].

Theorem 7. Let $f, g:[a, b] \rightarrow \mathbb{R}_{\mathcal{I}}^{+}$be two interval-valued functions such that $f=[f, \bar{f}], g=[\underline{g}, \bar{g}]$ and $f g \in \mathcal{I R}_{([a, b])}, h_{1}, h_{2}:[0,1] \rightarrow \mathbb{R}^{+}$be non-negative functions and $h_{1}\left(\frac{1}{2}\right) h_{2}\left(\frac{1}{2}\right) \neq 0$. If $\bar{f} \in$ $\operatorname{SHX}\left(h_{1},[a, b], \mathbb{R}_{\mathcal{I}}^{+}\right), g \in \operatorname{SHX}\left(h_{2},[a, b], \mathbb{R}_{\mathcal{I}}^{+}\right)$, then

$$
\begin{aligned}
& \frac{1}{\alpha h_{1}\left(\frac{1}{2}\right) h_{2}\left(\frac{1}{2}\right)} f\left(\frac{2 a b}{a+b}\right) g\left(\frac{2 a b}{a+b}\right) \\
\supseteq & \Gamma(\alpha)\left(\frac{a b}{b-a}\right)^{\alpha}\left[\mathcal{J}_{\left(\frac{1}{b}\right)^{+}}^{\alpha}(f \circ \xi)\left(\frac{1}{a}\right)(g \circ \xi)\left(\frac{1}{a}\right)+\mathcal{J}_{\left(\frac{1}{a}\right)^{-}}^{\alpha}(f \circ \xi)\left(\frac{1}{b}\right)(g \circ \xi)\left(\frac{1}{b}\right)\right] \\
& +\int_{0}^{1}\left[v^{\alpha-1}+(1-v)^{\alpha-1}\right]\left[h_{1}(v) h_{2}(1-v) \mathcal{M}(a, b)+h_{1}(v) h_{2}(v) \mathcal{N}(a, b)\right] d v
\end{aligned}
$$


where $\xi(x)=\frac{1}{x}$.

Proof. The proof is completed by combining Theorem 1, 4 and 5 .

Remark 7. If $h_{1}(v)=h_{2}(v)=v$, then

$$
\begin{aligned}
& 4 f\left(\frac{2 a b}{a+b}\right) g\left(\frac{2 a b}{a+b}\right) \\
\supseteq & \Gamma(\alpha+1)\left(\frac{a b}{b-a}\right)^{\alpha}\left[\mathcal{J}_{\left(\frac{1}{b}\right)^{+}}^{\alpha}(f \circ \xi)\left(\frac{1}{a}\right)(g \circ \xi)\left(\frac{1}{a}\right)+\mathcal{J}_{\left(\frac{1}{a}\right)^{-}}^{\alpha}(f \circ \xi)\left(\frac{1}{b}\right)(g \circ \xi)\left(\frac{1}{b}\right)\right] \\
& +\int_{0}^{1}\left[v^{\alpha-1}+(1-v)^{\alpha-1}\right]\left[v(1-v) \mathcal{M}(a, b)+v^{2} \mathcal{N}(a, b)\right] d v .
\end{aligned}
$$

If $\alpha=1$, then we get Theorem 4 of [15].

\section{Conclusions}

This paper proved the relationship between interval $h$-convex functions and interval harmonically $h$-convex functions. Further, we obtained some Hermite-Hadamard type inequalities for IVFs via interval Riemann-Liouville type fractional integrals. The results obtained in this article are the generalizations and refinements of the earlier works. Moreover, these results may be extended for other kinds of convex functions including interval $\left(h_{1}, h_{2}\right)$-convex functions and interval Log- $h$-convex functions and used as a method to establish the Hermite-Hadamard type inequalities for other types of interval harmonically convex functions. As a future research direction, we intend to investigate Hermite-Hadamard type inequalities for IVFs on time scales and some applications in interval optimization, probability, among others.

Author Contributions: Conceptualization, F.S., G.Y., D.Z., and W.L.; methodology, F.S., G.Y., D.Z., and W.L.; writing-original draft preparation, F.S.; writing-review and editing, F.S.; supervision, D.Z.; and W.L.; project administration, G.Y.; and funding acquisition, G.Y.; All authors have read and agreed to the published version of the manuscript.

Funding: This work was supported in part by the Fundamental Research Funds for Central Universities (2019B44914), Special Soft Science Research Projects of Technological Innovation in Hubei Province (2019ADC146), Key Projects of Educational Commission of Hubei Province of China (D20192501), the Natural Science Foundation of Jiangsu Province (BK20180500) and the National Key Research and Development Program of China (2018YFC1508100).

Acknowledgments: The authors are very grateful to three anonymous referees, for several valuable and helpful comments, suggestions and questions, which helped them to improve the paper into present form.

Conflicts of Interest: The authors declare no conflict of interest.

\section{References}

1. Bombardelli, M.; Varošanec, S. Properties of $h$-convex functions related to the Hermite-Hadamard-Fejér inequalities. Comput. Math. Appl. 2009, 58, 1869-1877. [CrossRef]

2. Dragomir, S.S. Inequalities of Hermite-Hadamard type for $h$-convex functions on linear spaces. Proyecciones 2015, 34, 323-341. [CrossRef]

3. Noor, M.A.; Noor, K.I.; Awan, M.U.; Costache, S. Some integral inequalities for harmonically h-convex functions. Politehn. Univ. Bucharest Sci. Bull. Ser. A Appl. Math. Phys. 2015, 77, 5-16.

4. Sarikaya, M.Z.; Saglam, A.; Yildirim, H. On some Hadamard-type inequalities for $h$-convex functions. J. Math. Inequal 2008, 2, 335-341. [CrossRef]

5. Sarikaya, M.Z.; Set, E.; Yaldiz, H.; Başak, N. Hermite-Hadamard's inequalities for fractional integrals and related fractional inequalities. Math. Comput. Model. 2013, 57, 2403-2407. [CrossRef]

6. Chen, F. Extensions of the Hermite-Hadamard inequality for harmonically convex functions via fractional integrals. Appl. Math. Comput. 2015, 268, 121-128. [CrossRef] 
7. Işcan, İ.; Wu, S.H. Hermite-Hadamard type inequalities for harmonically convex functions via fractional integrals. Appl. Math. Comput. 2014, 238, 237-244. [CrossRef]

8. Noor, M.A.; Noor, K.I.; Mihai, M.V.; Awan, M.U. Fractional Hermite-Hadamard inequalities for some classes of differentiable preinvex functions. Sci. Bull. Politeh. Univ. Buchar. Ser. A Appl. Math. Phys. 2016, 78, 163-174.

9. Snyder, J.M. Interval analysis for computer graphics. SIGGRAPH Comput. Graph 1992, 26, 121-130. [CrossRef]

10. Rothwell, E.J.; Cloud, M.J. Automatic error analysis using intervals. IEEE Trans. Ed. 2011, 55, 9-15. [CrossRef]

11. Chalco-Cano, Y.; Lodwick, W.A.; Condori-Equice, W. Ostrowski type inequalities for interval-valued functions using generalized Hukuhara derivative. Comput. Appl. Math. 2012, 31, 457-472.

12. Flores-Franulič, A.; Chalco-Cano, Y.; Román-Flores, H. An Ostrowski type inequality for interval-valued functions. In Proceedings of the 2013 Joint IFSA World Congress and NAFIPS Annual Meeting (IFSA/NAFIPS), Edmonton, AB, Canada, 24-28 June 2013; Volume 35, pp. 1459-1462.

13. Román-Flores, H.; Chalco-Cano, Y.; Lodwick, W.A. Some integral inequalities for interval-valued functions. Comput. Appl. Math. 2016, 35, 1-13. [CrossRef]

14. Zhao, D.F.; An, T.Q.; Ye, G.J.; Liu, W. New Jensen and Hermite-Hadamard type inequalities for $h$-convex interval-valued functions. J. Inequal. Appl. 2018, 2018, 302. [CrossRef]

15. Zhao, D.F.; An, T.Q.; Ye, G.J.; Torres, D.F.M. On Hermite-Hadamard type inequalities for harmonically h-convex interval-valued functions. Math. Inequal. Appl. 2020, 23, 95-105.

16. An, Y.R.; Ye, G.J.; Zhao, D.F.; Liu, W. Hermite-Hadamard Type Inequalities for Interval $(h 1, h 2)$-Convex Functions. Mathematics 2019, 7, 436. [CrossRef]

17. Büdak, H.; Tunc, T.; Sarikaya, M.Z. Fractional Hermite-Hadamard type inequalities for interval-valued functions. Proc. Amer. Math. Soc. 2020, 148, 705-718. [CrossRef]

18. Liu, X.L.; Ye, G.J.; Zhao, D.F.; Liu, W. Fractional Hermite-Hadamard type inequalities for interval-valued functions. J. Inequal. Appl. 2019, 2019, 266. [CrossRef]

19. Markov, S. Calculus for interval functions of a real variable. Computing 1979, 22, 325-337. [CrossRef]

20. Moore, R.E. Interval Analysis; Prentice-Hall: Englewood Cliffs, NJ, USA, 1966.

(c) 2020 by the authors. Licensee MDPI, Basel, Switzerland. This article is an open access article distributed under the terms and conditions of the Creative Commons Attribution (CC BY) license (http:/ / creativecommons.org/licenses/by/4.0/). 